\title{
Correction: Xu, S.; Li, Y.; Liu, Y.; Sun, J.; Ren, H.; Wu, S.-T. Fast-Response Liquid Crystal Microlens. Micromachines 2014, $5,300-324$
}

\section{Su Xu ${ }^{1}$, Yan Li ${ }^{2}$, Yifan Liu ${ }^{1}$, Jie Sun ${ }^{1}$, Hongwen Ren ${ }^{3}$ and Shin-Tson Wu ${ }^{1, *}$}

1 The College of Optics and Photonics (CREOL), University of Central Florida, 4000 Central Florida Blvd, Orlando, FL 32816, USA; E-Mail: suxu@creol.ucf.edu

2 National Engineering Lab for TFT-LCD Materials and Technologies, Department of Electronic Engineering, Shanghai Jiao Tong University, Shanghai 200240, China; E-Mail: yan.li@sjtu.edu.cn

3 Department of Polymer-Nano Science and Technology, Chonbuk National University, Jeonju, Jeonbuk 561-756, Korea; E-Mail: hongwen@jbnu.ac.kr

* Authors to whom correspondence should be addressed; E-Mail: swu@ucf.edu; Tel.: +1-407-823-4763; Fax: +1-407-823-6880.

Academic Editor: Joost Lötters

Received: 9 February 2015 / Accepted: 18 February 2015 / Published: 2 March 2015

We have found three errors in our paper [1], and thus would like to make the following corrections to this paper:

On page 302, in the second paragraph, line 12, "tenabiliby" should be changed to "tunability".

On page 302, in the fourth paragraph, "In a typical cell, LC passing the cell, where $d$ is the cell gap. When a sufficiently high voltage is applied to the indium tin oxide (ITO) electrodes, the LC directors will be reoriented material is sandwiched between two substrates coated with electrodes (e.g., indium tin oxide, ITO) and surface alignment layers (e.g., polyimide, PI) [53]" should be changed to "In a typical cell, LC material is sandwiched between two substrates coated with electrodes (e.g., indium tin oxide, ITO) and surface alignment layers (e.g., polyimide, PI) [53]".

On page 303, in the first paragraph, "It will experience an optical path of $L=d n$ e after in vertical direction and the optical path becomes $L=d n_{\mathrm{o}}$ (Figure 1b)" should be changed to "It will experience an optical path of $L=d n_{\mathrm{e}}$ after passing the cell, where $d$ is the cell gap. When a sufficiently high voltage is applied to the ITO electrodes, the LC directors will be reoriented in vertical direction and the optical path becomes $L=d n_{\mathrm{o}}$ (Figure 1b)." 
The Editorial Office would like to apologize for any inconvenience caused to the readers by these changes.

The corrected version can be accessed at: http://www.mdpi.com/2072-666X/6/3/328/s1.

\section{Reference}

1. Xu, S.; Li, Y.; Liu, Y.; Sun, J.; Ren, H.; Wu, S.-T. Fast-Response Liquid Crystal Microlens. Micromachines 2014, 5, 300-324.

(C) 2015 by the authors; licensee MDPI, Basel, Switzerland. This article is an open access article distributed under the terms and conditions of the Creative Commons Attribution license (http://creativecommons.org/licenses/by/4.0/). 